Article

\title{
Comparison of Chemical Compositions in Pseudostellariae Radix from Different Cultivated Fields and Germplasms by NMR-Based Metabolomics
}

\author{
Yujiao Hua ${ }^{\dagger}$, Ya Hou ${ }^{\dagger}$, Shengnan Wang, Yang Ma, Zixiu Liu, Lisi Zou, Xunhong Liu *, \\ Yiyuan Luo and Juanxiu Liu \\ College of Pharmacy, Nanjing University of Chinese Medicine, Nanjing 210023, China; \\ huayujiao1020@163.com (Y.H.); yp0317@163.com (Y.H.); jshmwsn@163.com (S.W.); \\ nzymayang1990@163.com (Y.M.); liuzixiu3221@126.com (Z.L.); ggn0117@sohu.com (L.Z.); \\ luoyiyuan0012@sohu.com (Y.L.); liujx0516@163.com (J.L.) \\ * Correspondence: liuxunh1959@sohu.com; Tel./Fax: +86-25-8581-1524 \\ + These authors contributed equally to this work. \\ Academic Editor: Derek J. McPhee \\ Received: 31 August 2016; Accepted: 9 November 2016; Published: 15 November 2016
}

\begin{abstract}
Pseudostellariae Radix (PR) is an important traditional Chinese medicine (TCM), which is consumed commonly for its positive health effects. However, the chemical differences of PR from different cultivated fields and germplasms are still unknown. In order to comprehensively compare the chemical compositions of PR from different cultivated fields, in this study, ${ }^{1} \mathrm{H}-\mathrm{NMR}$-based metabolomics coupled with high performance liquid chromatography (HPLC) were used to investigate the different metabolites in PR from five germplasms (jr, zs1, zs2, sb, and xc) cultivated in traditional fields (Jurong, Jiangsu, JSJR) and cultivated fields (Zherong, Fujian, FJZR). A total of 34 metabolites were identified based on ${ }^{1} \mathrm{H}-\mathrm{NMR}$ data, and fourteen of them were found to be different in PR from JSJR and FJZR. The relative contents of alanine, lactate, lysine, taurine, sucrose, tyrosine, linolenic acid, $\gamma$-aminobutyrate, and hyperoside in PR from JSJR were higher than that in PR from FJZR, while PR from FJZR contained higher levels of glutamine, raffinose, xylose, unsaturated fatty acid, and formic acid. The contents of Heterophyllin A and Heterophyllin B were higher in PR from FJZR. This study will provide the basic information for exploring the influence law of ecological environment and germplasm genetic variation on metabolite biosynthesis of PR and its quality formation mechanism.
\end{abstract}

Keywords: Pseudostellariae Radix; ${ }^{1} \mathrm{H}-\mathrm{NMR}$; metabolomics; cultivated fields; germplasms

\section{Introduction}

Pseudostellariae Radix (Taizishen in Chinese, PR) is an arid tuberous root of Pseudostellaria heterophylla (Miq.) Pax ex Pax et Hoffm (Caryophyllaceae). It is a type of staple traditional Chinese medicine (TCM) which has the functions of strengthening the spleen, replenishing Qi, moistening lungs, and producing fluids. This medicinal herb is consumed clinically for its positive effects [1]. It is reported that PR can be used for inappetence, thirst, debility, diabetes, and weakness after illness, and it has become an important medicine to cure loss of appetite in children due to spleen deficiency [2-6]. As the resources of wild PR are declining and the demand for original medicinal materials is rising annually, the government has established a large-scale cultivation base for PR in Zherong City, Fujian Province; Shibing City, Guizhou Province; and Xuanzhou City, Anhui Province, China, in addition to the traditional growing field in Jurong City, Jiangsu Province. However, due to differences in the 
ecological environments and expanding cultivated fields, the accumulation of active components and the quality of the material have shown marked differences in PR from various sites. For example, Heterophyllin A and Heterophyllin B were the active components in PR, which showed remarkable differences in different sites, and this may lead to differences in quality of commercial medicinal materials [7]. Thus, it is difficult to implement commodity standardization of medicinal materials and to ensure the effectiveness of its clinical application. Thus, how to ensure the quality and uniformity of PR have become key issues in its production process.

It has been reported that nucleoside, polysaccharide, saponin, pseudostellarins, and heterophyllins are rich in PR [8-11]. Currently, the quality assessment of PR are mainly focused on the quantitative determination of nucleoside, polysaccharide, saponin, pseudostellarins, and heterophyllins. In Chinese Pharmacopoeia 2010, the content of heterophyllins B was used to assess the quality of PR. However, the components of PR are complex, one or several components cannot assess the quality of this herbal medicine. Metabolomics has recently emerged as an important method for modern research on medicinal plants, and this technology can analyze the whole components in PR, which may fully reflect the immanent quality of medicinal materials. NMR spectroscopy is a key technique used for plant metabolomics due to its non-selectivity, speed, high throughout and relatively easier sample preparation [12]. The ${ }^{1} \mathrm{H}$ NMR-based metabolomics approach has been widely applied to reveal metabolic differences among herbal medicines, such as Forsythia suspense [13], Coptis chinensis [14], Panax ginseng [15]. In the present study, ${ }^{1} \mathrm{H}-\mathrm{NMR}$-based metabolomics coupled with HPLC quantitative determination were performed to compare the chemical differences and find the change regularity in PR from different cultivated fields and germplasms. The results may provide the basic information for exploring the influence law of ecological environment and germplasm genetic variation on metabolite biosynthesis of PR and its quality formation mechanism.

\section{Results and Discussion}

\section{1. ${ }^{1} \mathrm{H}-\mathrm{NMR}$ Metabolic Profiling}

\subsubsection{Assignment of ${ }^{1} \mathrm{H}-\mathrm{NMR}$ Spectra}

${ }^{1} \mathrm{H}-\mathrm{NMR}$ spectra signals were assigned based on comparisons with the chemical shift of authentic standards, metabolites in the Biological Magnetic Resonance Data Bank (BMRB) and NMR data in the literature $[16,17]$. Representative ${ }^{1} \mathrm{H}-\mathrm{NMR}$ spectra of $\mathrm{PR}$ from different cultivated fields were shown in Figures 1 and 2, with metabolites indicated based on their chemical shifts, coupling constants, and peak pattern. A total of 34 metabolites were identified in Table 1 . The atlas can be divided into three regions, a high field region ( $\delta 3.10-0.00)$, consisting of amino acids and organic acids, such as leucine, lysine, alanine, succinate, and 2-Ketoisovaleric acid etc.; a middle field region ( $\delta 6.00-3.10)$, mainly including carbohydrates, such as $\alpha$-glucose, sucrose, raffinose, and xylose etc.; and a low field region ( $\delta 10.00-6.00)$ including fumaric acid, formic acid, quercetin, hyperoside, and luteolin, etc.

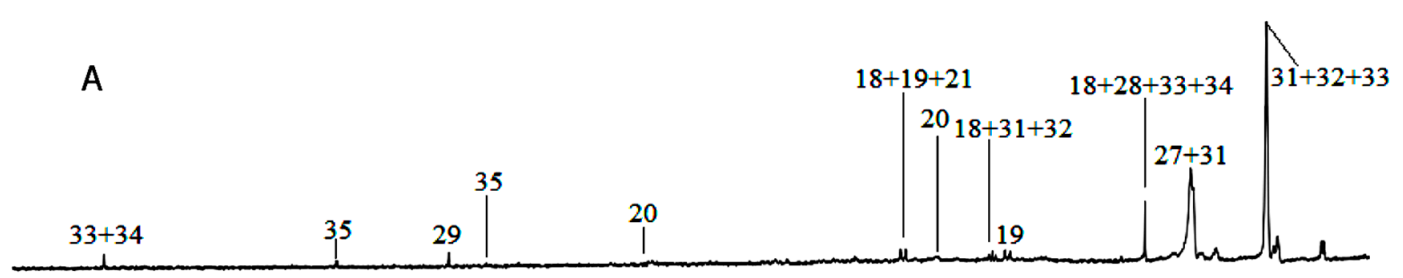

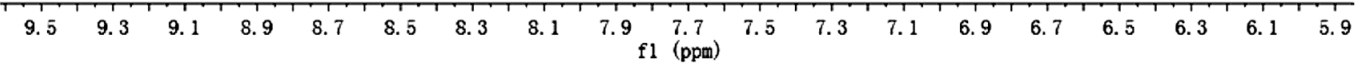

Figure 1. Cont. 

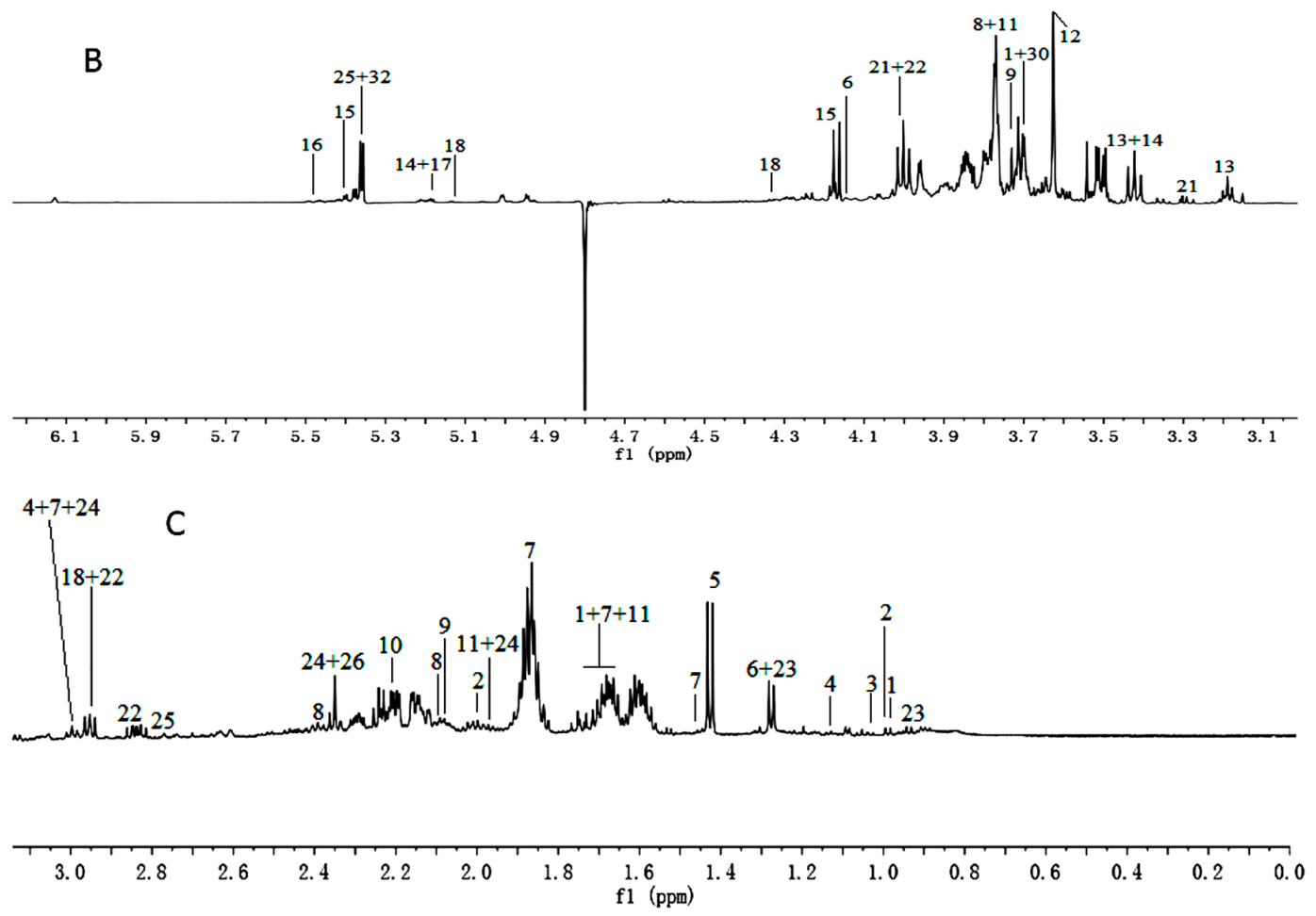

Figure 1. ${ }^{1} \mathrm{H}-\mathrm{NMR}$ spectra $(600 \mathrm{MHz})$ of aqueous methanol fractions in PR from JSJR in low field region (A); middle field region (B) and high field region (C). NMR solvent: $\mathrm{CD}_{3} \mathrm{OD}: \mathrm{KH}_{2} \mathrm{PO}_{4}$ buffer in $\mathrm{D}_{2} \mathrm{O}$ 1:1.
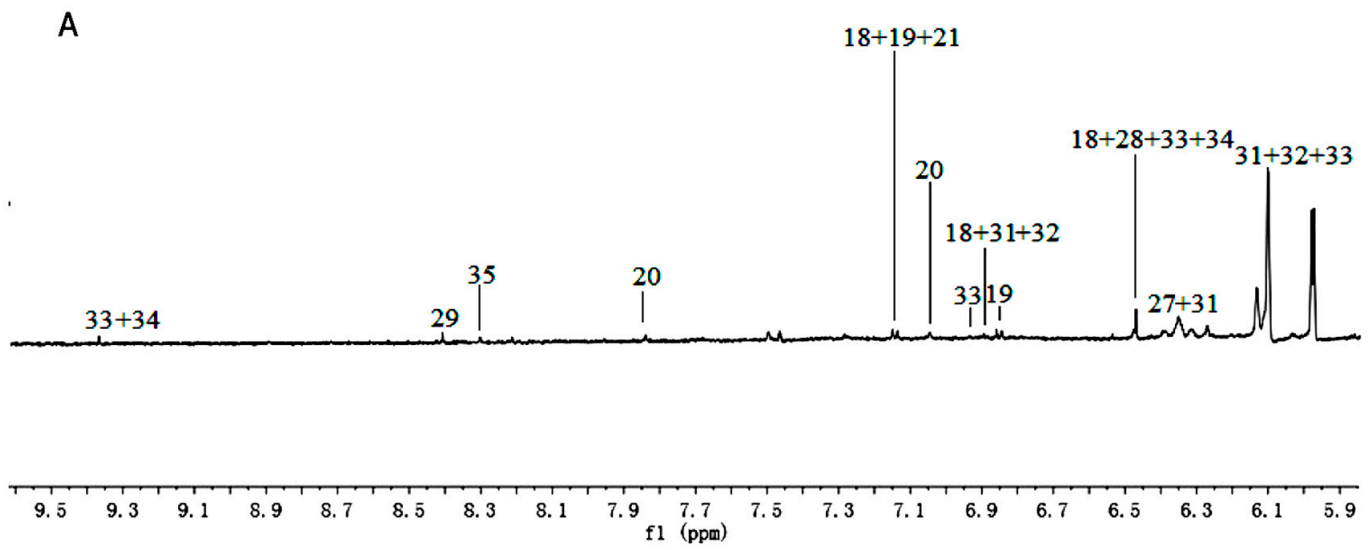

B

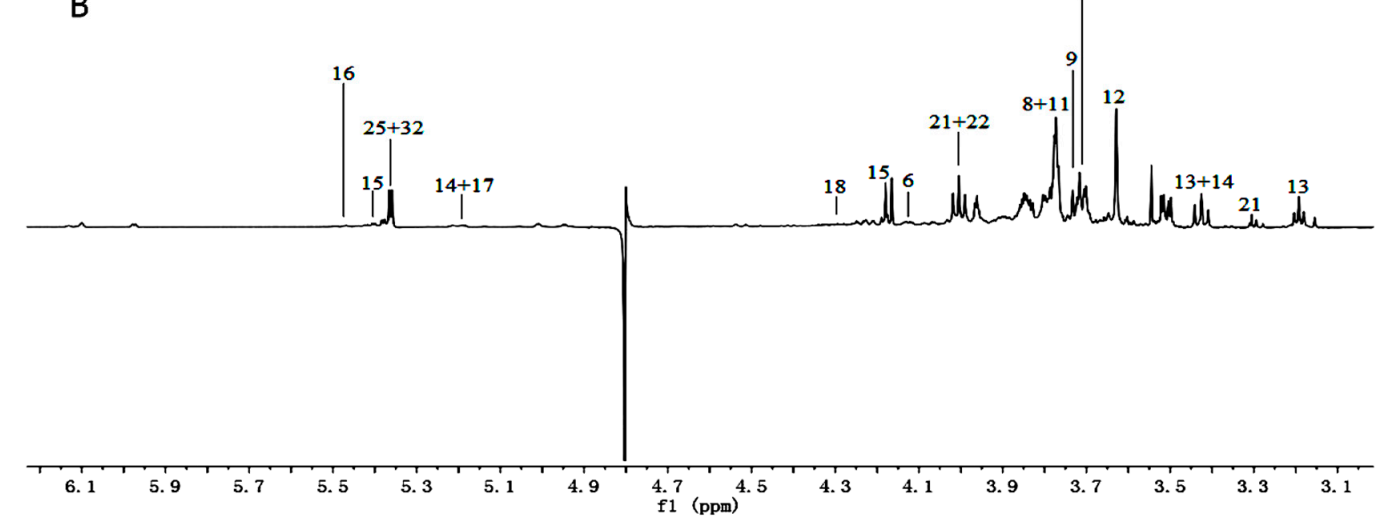

Figure 2. Cont. 

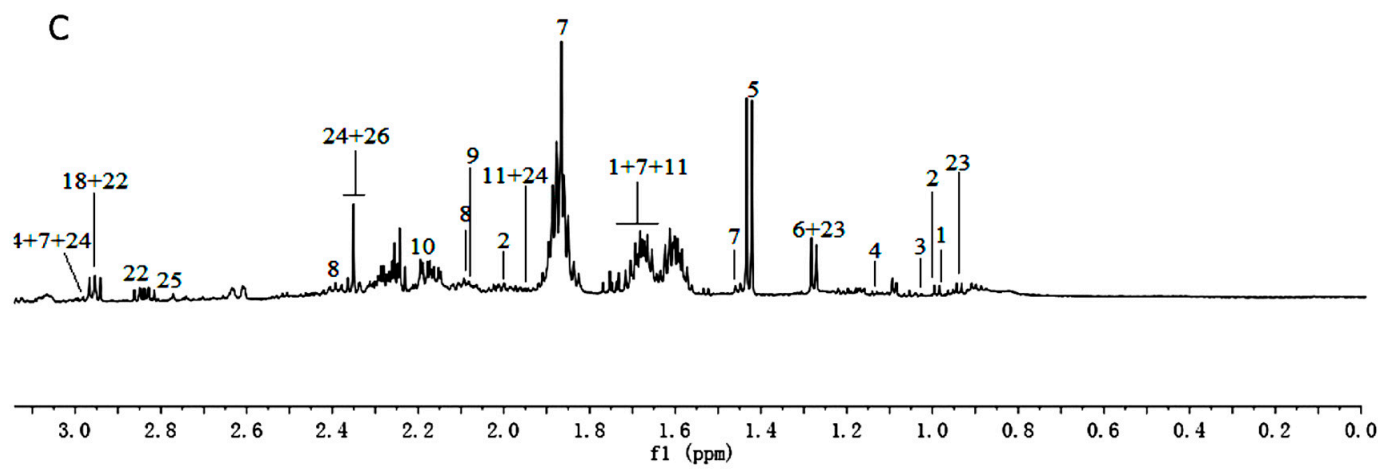

Figure 2. ${ }^{1} \mathrm{H}-\mathrm{NMR}$ spectra $(600 \mathrm{MHz})$ of aqueous methanol fractions in PR from FJZR in low field region (A); middle field region (B) and high field region $(\mathbf{C})$. NMR solvent: $\mathrm{CD}_{3} \mathrm{OD}: \mathrm{KH}_{2} \mathrm{PO}_{4}$ buffer in $\mathrm{D}_{2} \mathrm{O} 1: 1$.

Table 1. Metabolites detected by ${ }^{1} \mathrm{H}-\mathrm{NMR}$ in PR from different cultivated fields.

\begin{tabular}{|c|c|c|c|c|}
\hline No. & Metabolites & $\begin{array}{l}\text { Chemical Shift for Standards }(\delta, \mathrm{ppm}) \\
\text { Coupling Constants }(J, \mathrm{~Hz})\end{array}$ & $\begin{array}{l}\text { Chemical Shift }(\delta, \text { ppm), Coupling } \\
\text { Constants }(J, \mathrm{~Hz})\end{array}$ & VIP \\
\hline 1 & Leucine & $0.95(\mathrm{t}, 5.9), 1.72(\mathrm{~m})$ & $0.97(\mathrm{~d}, 7.1), 1.72(\mathrm{~m}), 3.71(\mathrm{~s})$ & 0.32 \\
\hline 2 & Isoleucine & $\begin{array}{l}0.926(\mathrm{t}, 7.414), 0.997(\mathrm{~d}, 7.001), 1.248(\mathrm{~m}) \\
\quad 1.457(\mathrm{~m}), 1.968(\mathrm{~m}), 3.66(\mathrm{~d}, 3.969)\end{array}$ & $1.02(\mathrm{t}, 7.6), 1.97(\mathrm{~m})$ & 1.09 \\
\hline 3 & Valine & $\begin{array}{c}0.976(\mathrm{~d}, 7.01), 1.029(\mathrm{~d}, 7.05), 2.261(\mathrm{~m}) \\
3.601(\mathrm{~d}, 4.33)\end{array}$ & $1.05(\mathrm{~d}, 6.8)$ & 1.17 \\
\hline 4 & 2-Ketoisovaleric acid & $1.15(\mathrm{~m}), 3.02(\mathrm{~m})$ & $1.13(\mathrm{~d}, 7.2), 3.03(\mathrm{~m})$ & 1.02 \\
\hline 5 & Alanine & $1.47(\mathrm{~d}, 7.2), 3.8(\mathrm{~m})$ & $1.43(\mathrm{~d}, 7.3)$ & 1.38 \\
\hline 6 & Lactate & $4.1(\mathrm{~s}), 1.3(\mathrm{~s})$ & $1.26(\mathrm{~s}), 4.10(\mathrm{~s})$ & 1.01 \\
\hline 7 & Lysine & $1.46(\mathrm{~m}), 1.7(\mathrm{~m}), 1.89(\mathrm{~m}), 3.02(\mathrm{t}, 6.09)$ & $1.44(\mathrm{~m}), 1.72(\mathrm{~m}), 1.87(\mathrm{~m}), 3.04(\mathrm{t}, 6.6)$ & 1.31 \\
\hline 8 & Glutamine & $2.12(\mathrm{~m}), 2.45(\mathrm{~m}), 3.76(\mathrm{t}, 6.18)$ & $2.12(\mathrm{~m}), 2.37(\mathrm{~m}), 3.76(\mathrm{~m})$ & 1.61 \\
\hline 9 & Glutamate & $2.04(\mathrm{~m}), 3.75(\mathrm{dd}, 7.186,4.72), 2.34(\mathrm{~m})$ & $2.07(\mathrm{~m}), 2.34(\mathrm{~m}), 3.74(\mathrm{~m})$ & 0.11 \\
\hline 10 & Acetoacetate & $2.27(\mathrm{~s}), 3.43(\mathrm{~s})$ & $2.22(\mathrm{~m})$ & 1.51 \\
\hline 11 & Arginine & $1.68(\mathrm{~m}), 1.90(\mathrm{~m}), 3.22(\mathrm{t}, 6.93), 3.76(\mathrm{t}, 6.11)$ & $1.72(\mathrm{~m}), 1.92(\mathrm{~m}), 3.75(\mathrm{t}, 6.1)$ & 1.39 \\
\hline 12 & Glycine & $3.54(\mathrm{~s})$ & $3.61(\mathrm{~s})$ & 1.47 \\
\hline 13 & Taurine & $3.25(\mathrm{t}, 6.57), 3.42(\mathrm{t}, 6.62)$ & $3.40(\mathrm{t}, 12.0), 3.27(\mathrm{t}, 10.2)$ & 1.06 \\
\hline 14 & $\alpha$-Glucose & $5.2(\mathrm{~d}, 3.7)$ & $3.40(\mathrm{~m}), 5.19(\mathrm{~d}, 3.7)$ & 0.36 \\
\hline 15 & Sucrose & $5.42(\mathrm{~d}, 3.6), 4.19(\mathrm{~d}, 8.4)$ & $5.40(\mathrm{~d}, 3.9), 4.18(\mathrm{~d}, 3.9)$ & 1.53 \\
\hline 16 & Raffinose & $4.97(\mathrm{~d}, 3.6), 5.43(\mathrm{~d}, 4.2)$ & $5.49(\mathrm{~d}, 3.8)$ & 1.33 \\
\hline 17 & Xylose & $4.55(\mathrm{~d}, 9)$ & $5.19(\mathrm{~m})$ & 0.36 \\
\hline 18 & Salvianolic acid B & $7.15(\mathrm{~d}, 8.8), 6.80(\mathrm{~d}, 7.2), 6.50(\mathrm{~d}, 2.8), 2.95(\mathrm{~m})$ & $\begin{array}{l}7.14(\mathrm{~d}, 8.2), 6.85(\mathrm{~d}, 8.6), 6.47(\mathrm{~d}, 1.8) \\
5.13(\mathrm{~m}), 4.29(\mathrm{~d}, 4.8), 2.94(\mathrm{~m})\end{array}$ & 1.05 \\
\hline 19 & Tyrosine & $6.83(\mathrm{~d}, 8.0), 7.15(\mathrm{~m}), 6.87(\mathrm{~m}), 7.17(\mathrm{~m})$ & $7.12(\mathrm{t}, 8.4), 6.83(\mathrm{t}, 6.6)$ & 1.35 \\
\hline 20 & Histidine & $7.09(\mathrm{~d}, 0.58), 7.9(\mathrm{~d}, 1.13)$ & $7.10(\mathrm{~d}, 8.4), 7.90(\mathrm{~m})$ & 0.89 \\
\hline 21 & Phenylalanine & $\begin{array}{c}3.19(\mathrm{~m}), 3.98(\mathrm{dd}, 7.88,5.31), 7.32(\mathrm{~d}, 6.96) \\
7.36(\mathrm{~m}), 7.42(\mathrm{~m})\end{array}$ & $3.28(\mathrm{~m}), 3.99(\mathrm{~m}), 7.14(\mathrm{~d}, 8.2)$ & 1.53 \\
\hline 22 & Asparagine & $2.94(\mathrm{~m}), 2.84(\mathrm{~m}), 4.00(\mathrm{dd}, 7.69,4.2)$ & $\begin{array}{c}2.88(\mathrm{dd}, 16.1,7.6), 2.96(\mathrm{dd}, 16.1,7.6) \\
4.00(\mathrm{~m})\end{array}$ & 0.52 \\
\hline 23 & Linolenic acid & $0.98(t, 9.8)$ & $0.96(\mathrm{t}, 6.0), 1.30(\mathrm{brs})$ & 1.07 \\
\hline 24 & $\gamma$-aminobutyrate & $1.94(\mathrm{~m}), 2.48(\mathrm{t}, 7.36), 3.03(\mathrm{t}, 7.58)$ & $1.92(\mathrm{~m}), 2.32(\mathrm{t}, 9.6), 3.04(\mathrm{t}, 6.6)$ & 1.35 \\
\hline 25 & Unsaturated fatty acid & $2.85(\mathrm{~m}), 5.30(\mathrm{~m})$ & $2.75(\mathrm{~m}), 5.34(\mathrm{~m})$ & 1.06 \\
\hline 26 & Succinate & $2.39(\mathrm{~s})$ & $2.32(\mathrm{~s})$ & 0.81 \\
\hline 27 & Ferulic acid & $7.15(\mathrm{~d}, 8.4), 6.32(\mathrm{~m})$ & $7.14(\mathrm{~d}, 8.2), 6.34(\mathrm{~m})$ & 1.53 \\
\hline 28 & Fumaric acid & $6.55(\mathrm{~s})$ & $6.47(\mathrm{~s})$ & 1.69 \\
\hline 29 & Formic acid & $8.32(\mathrm{~s})$ & $8.40(\mathrm{~s})$ & 1.43 \\
\hline 30 & Dimethylglycine & $2.95(\mathrm{~s}), 3.75(\mathrm{~s})$ & $2.94(\mathrm{~s}), 3.71(\mathrm{~s})$ & 1.12 \\
\hline 31 & Quercetin & $\begin{array}{c}6.18(\mathrm{~d}, 2), 6.39(\mathrm{~d}, 2), 6.88(\mathrm{~d}, 8.5) \\
7.52(\mathrm{dd}, 2.2,8.5), 7.66(\mathrm{~d}, 2.2), 12.4(\mathrm{~s})\end{array}$ & $6.85(\mathrm{~d}, 8.6), 6.34(\mathrm{~d}, 4.9), 6.13(\mathrm{~d}, 1.8)$ & 1.14 \\
\hline 32 & Hyperoside & $6.90(\mathrm{~d}, 7.2), 5.40(\mathrm{~d}, 4.2)$ & $6.85(\mathrm{~d}, 8.6), 6.13(\mathrm{~d}, 1.6), 5.36(\mathrm{~d}, 3.9)$ & 1.14 \\
\hline 33 & Luteolin & $\begin{array}{c}6.4(\mathrm{~d}, 2), 6.68(\mathrm{~s}), 6.76(\mathrm{~d}, 2), 6.89(\mathrm{~d}, 9), 7.39(\mathrm{~d}, 2) \\
7.41(\mathrm{dd}, 2.2,9.0)\end{array}$ & $6.89(\mathrm{~d}, 8.6)$ & 1.32 \\
\hline 34 & Kaempferol & $8.05(\mathrm{~m}), 6.5(\mathrm{~d}, 2.0), 6.98(\mathrm{~m}), 6.25(\mathrm{~d}, 2.0)$ & $6.90(d, 9.0), 6.47(d, 1.2)$ & 1.69 \\
\hline
\end{tabular}




\subsubsection{Multivariate Data Analysis}

Based on the assignments of ${ }^{1} \mathrm{H}-\mathrm{NMR}$ spectra, chemical classification of all samples was performed by multivariate data analysis, which aimed to highlight the differences in PR from different cultivated fields. An unsupervised approach consisting of principal component analysis (PCA), a nonparametric method of classification, was used to reduce the dimensions of multivariate problems. The PCA score plot (PC1: $68.9 \%$, PC2: 17.8\%), which accounted for $86.7 \%$ of total variance of the dataset, showed clear separation in PR from different cultivated fields (Figure 3A). This indicated that the PR from JSJR and FJZR were significantly different in their metabolites, which may be caused by the ecological environment in different cultivated fields. However, the PR samples also showed interclass differences due to the different germplasms, which indicated that the genetic factors can affect the chemical components.
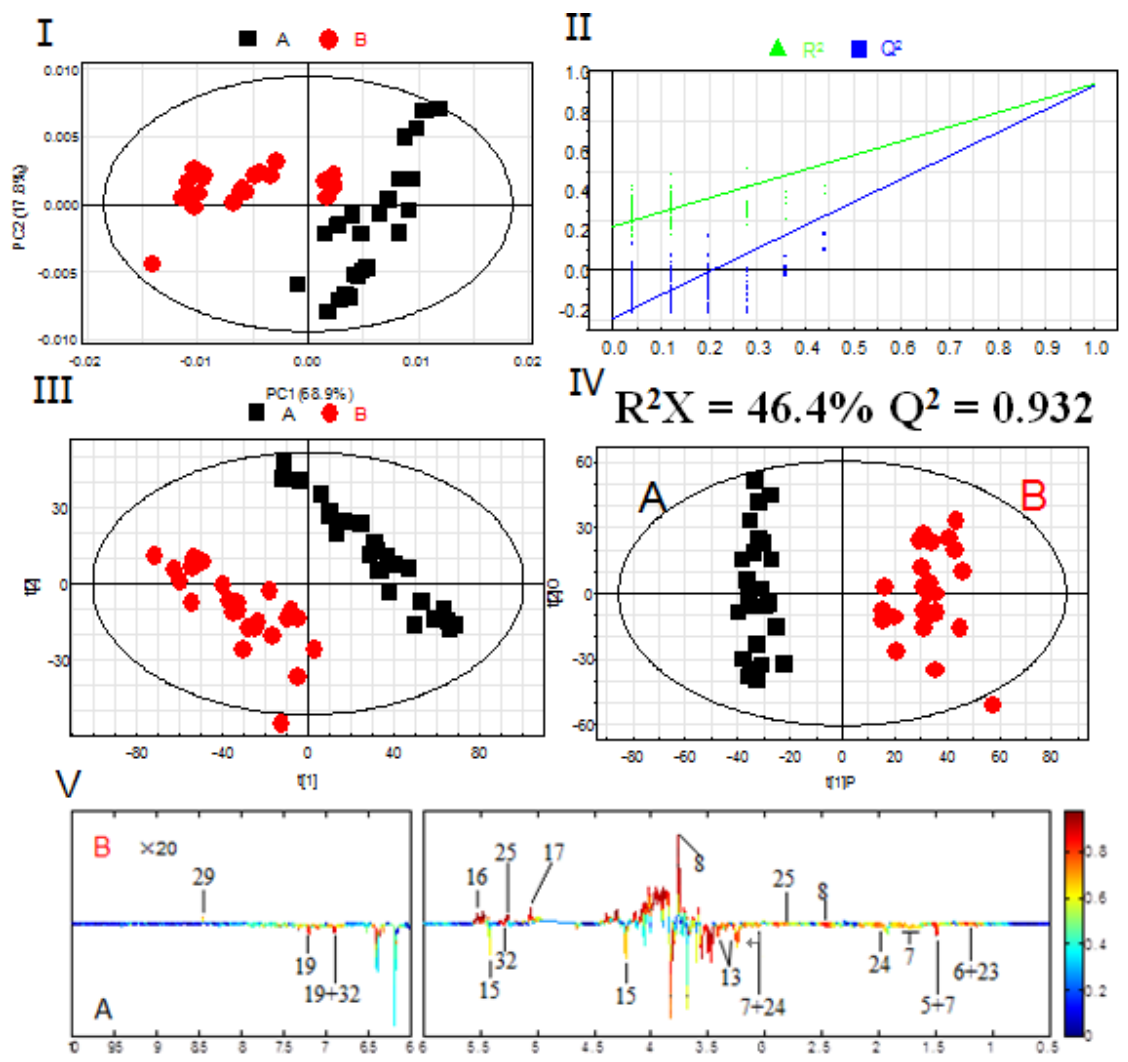

Figure 3. PCA scores plot (I); permutation test with 200 permutations of PLS-DA model (II); PLS-DA scores plot (III); OPLS-DA scores plot (IV); correlation coefficients loading plot (V) obtained from ${ }^{1} \mathrm{H}-\mathrm{NMR}$ metabolic profiles derived from aqueous methanol fractions of PR from different cultivation fields. (A) JSJR; (B) FJZR.

The partial least square discriminant analysis (PLS-DA) extends a regression of PCA and uses class information to maximize the separation between groups of observations. This frequently used classification method is categorical (categories described with dummy variables) and expresses the class membership of the statistical units. In this study, PLS-DA model was also validated by a permutation test with 200 permutations (Figure 3B). The PLS-DA score plot $\left(R^{2} X=46.4 \%, R^{2} Y=0.947, Q^{2}=0.930\right.$, Figure $3 \mathrm{C}$ ) showed that the PR from JSJR and FJZR were clearly clustered into two groups, and the interclass differences were less than that in the PCA model, which was more useful to confirm the differences in two different groups.

To further identify the significant metabolites contributing to distinction in PR from two different cultivated fields, orthogonal partial least square discriminate analysis (OPLS-DA) of these ${ }^{1} \mathrm{H}-\mathrm{NMR}$ 
data was further performed (Figure 3D). Correlation coefficient can directly demonstrate the significantly differential metabolites in PR from different cultivated fields, the plus and minus represented the positive and negative relationship of metabolites. A total of 14 significantly differential metabolites were identified in PR from JSJR and FJZR (Table 2). According to the correlation coefficients loading plot (Figure 3E), the relative contents of alanine, lactate, lysine, taurine, sucrose, tyrosine, linolenic acid, $\gamma$-aminobutyrate, and hyperoside in PR from JSJR were higher than that in PR from FJZR. On the contrary, the relative contents of glutamine, raffinose, xylose, unsaturated fatty acid, and formic acid in PR from FJZR were much higher. This indicated that the ecological factor could be an important factor to affect the whole chemical components in the metabolomics of PR. To further demonstrate the ecological factor was the leading factor, the PCA model was used to analyze the PR from jr and zs germplasms which were cultivated in different fields (Jurong City, Jiangsu Province; Zherong City, Fujian Province; Shibing City, Guizhou Province; Xuancheng City, Anhui Province). The results showed that the germplasms had little influence on the whole chemical components in metabolomics of PR (Figure 4).

In fourteen significantly differential components, alanine, sucrose, and linolenic acid were known stress-responsive metabolites. Alanine accumulation in plants in response to exposure to a variety of stress conditions, including sinusoidally-varying magnetic fields (SVMF), is a general phenomenon. The research proposed that alanine is a universal first stress signal expressed by cells [18]. Sucrose is the main form of assimilated carbon which is produced during photosynthesis and then transported from source to sink tissues via the phloem of plants. Additionally, sucrose is engaged in plant defense by activating plant immune responses against pathogens [19]. Linolenic acid is associated with low temperature stress. The study found that the plant stored in $0{ }^{\circ} \mathrm{C}-$ Air and $0{ }^{\circ} \mathrm{C}-\mathrm{CA}$ had much higher degree of linolenic acid content in the plasma membrane than it did that in $5^{\circ} \mathrm{C}-\operatorname{Air}$ [20].

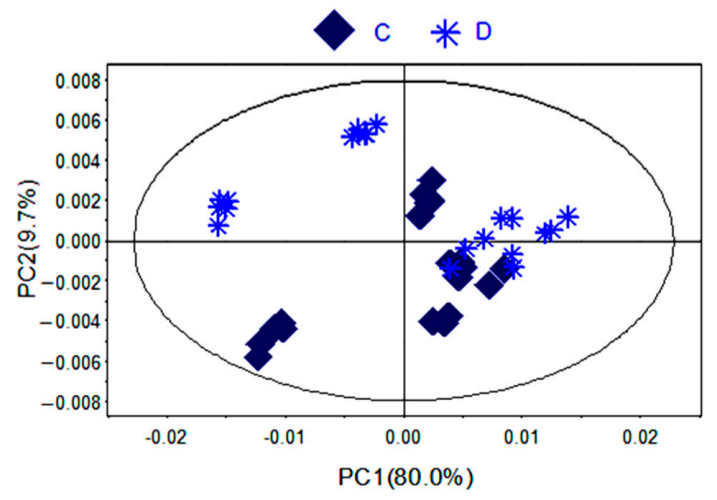

Figure 4. PCA scores plot of PR from different germplasms (C) zs; (D) jr.

Table 2. Significant differences in the chemical compositions and the correlation coefficient.

\begin{tabular}{ccc}
\hline No. & Metabolites & Correlation Coefficient \\
\hline 5 & Alanine & -0.893 \\
6 & Lactate & -0.865 \\
7 & Lysine & -0.648 \\
8 & Glutamine & +0.924 \\
13 & Taurine & -0.805 \\
15 & Sucrose & -0.645 \\
16 & Raffinose & +0.950 \\
17 & Xylose & +0.858 \\
19 & Tyrosine & -0.690 \\
23 & Linolenic acid & -0.589 \\
24 & $\gamma$-Aminobutyrate & -0.631 \\
25 & Unsaturated fatty acid & +0.707 \\
29 & Formic acid & +0.674 \\
32 & Hyperoside & -0.677 \\
\hline
\end{tabular}




\subsection{Content Determination of Heterophyllin $A$ and Heterophyllin $B$}

Heterophyllin A (HA) and Heterophyllin B (HB) are the characteristic components in PR. In Chinese Pharmacopoeia 2010, HB was the index component to assess the quality of PR. In this study, the ${ }^{1} \mathrm{H}-\mathrm{NMR}$ spectra has not detected these two components; thus, the contents of HA and HB in PR were determined by high performance liquid chromatography (HPLC). The HPLC chromatograms of mixed standards were in Figure 5 and PR samples were in Figure 6. The contents of HA and HB of PR in different cultivated fields were in Table 3. The results showed that the contents of HA and HB in PR from FJZR were much higher than the contents of HA and HB in JSJR. The contents of HB in PR from zs1 and zs2 germplasms were significantly lower than the contents of HB in PR from jr, sb, and xc germplasms; the contents of HA in PR from zs1 and zs2 germplasms were higher than the contents of HA in PR from jr, sb, and xc germplasms. The contents of HA were higher than the contents of HB in PR from zs1 and zs2 germplasms, the contents of $\mathrm{HB}$ were higher than the contents of HA in PR from $\mathrm{jr}, \mathrm{sb}$, and $\mathrm{xc}$ germplasms. This indicated that the genetic factor of germplasms may play an important role in heterophyllins of PR.

Table 3. Contents of Heterophyllin A and Heterophyllin B of PR in different cultivated fields. $\mathrm{Mg}^{-} \mathrm{g}^{-1}, n=3$.

\begin{tabular}{ccc}
\hline No. & Heterophyllin A & Heterophyllin B \\
\hline JSJR-jr & 9.32 & 138.42 \\
JSJR-zs1 & 36.32 & 2.54 \\
JSJR-zs2 & 16.22 & 3.16 \\
JSJR-sb & 6.88 & 65.47 \\
JSJR-xc & 6.20 & 130.69 \\
FJZR-jr & 14.99 & 175.58 \\
FJZR-zs1 & 43.73 & 2.75 \\
FJZR-zs2 & 28.09 & 5.02 \\
FJZR-sb & 12.18 & 96.01 \\
FJZR-xc & 11.55 & 149.27 \\
\hline
\end{tabular}

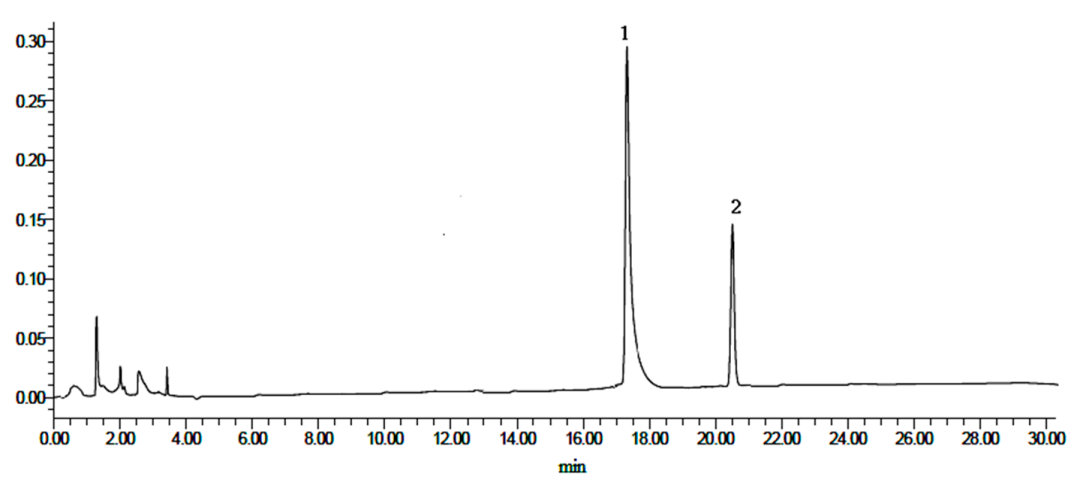

Figure 5. HPLC chromatograms of mixed standards. (1) Heterophyllin B, and (2) heterophyllin A.

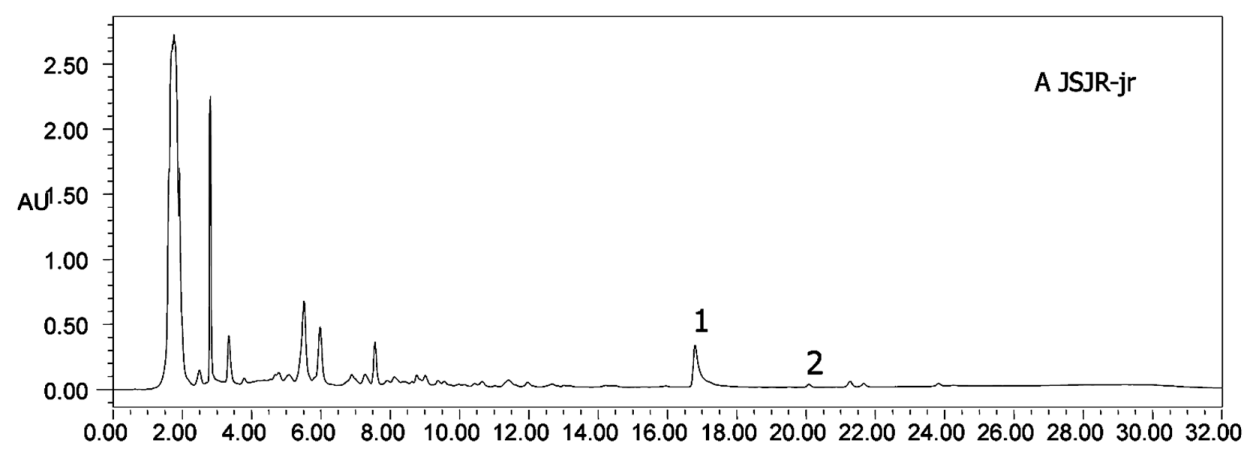

Figure 6. Cont. 

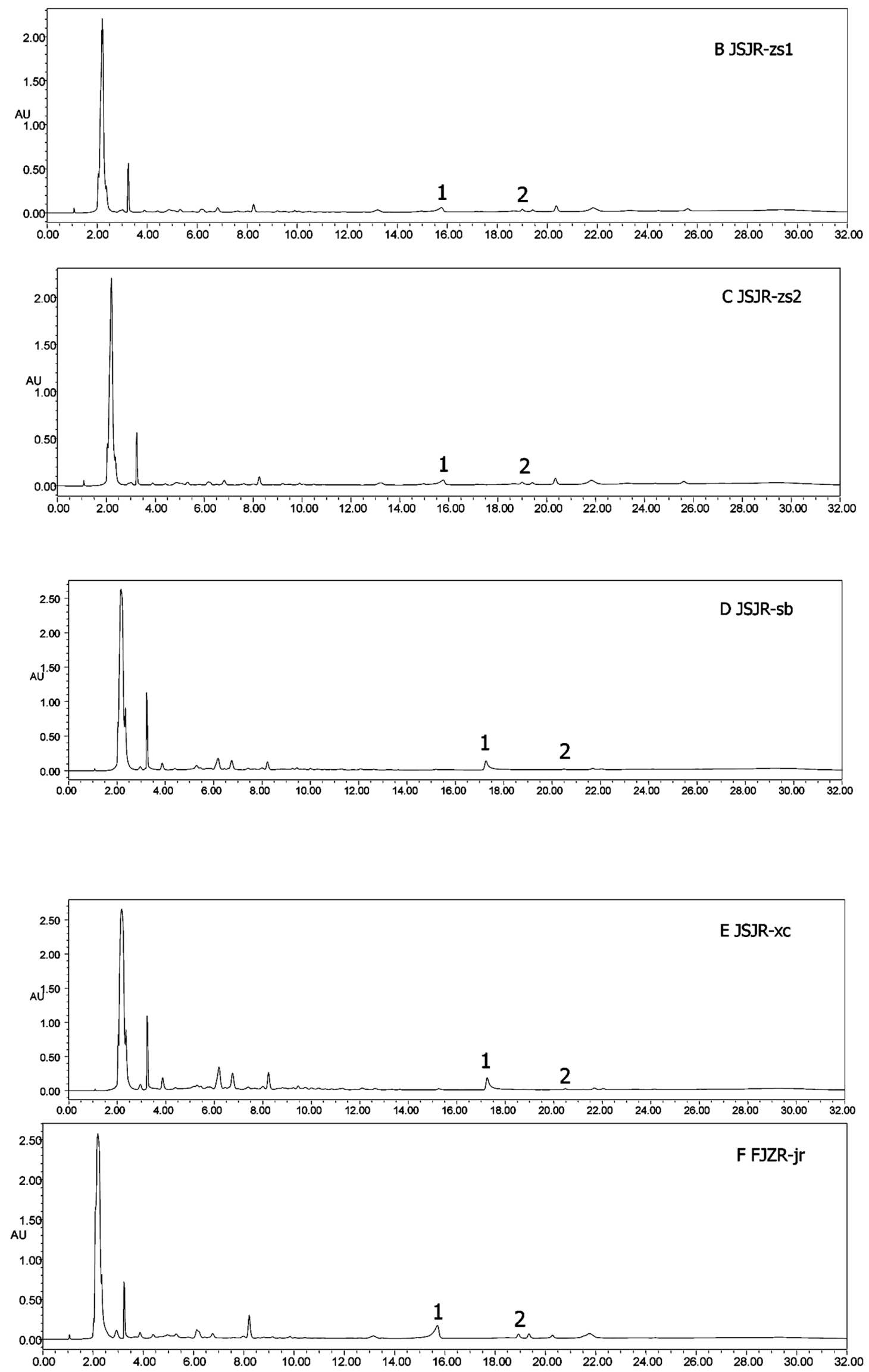

Figure 6. Cont. 

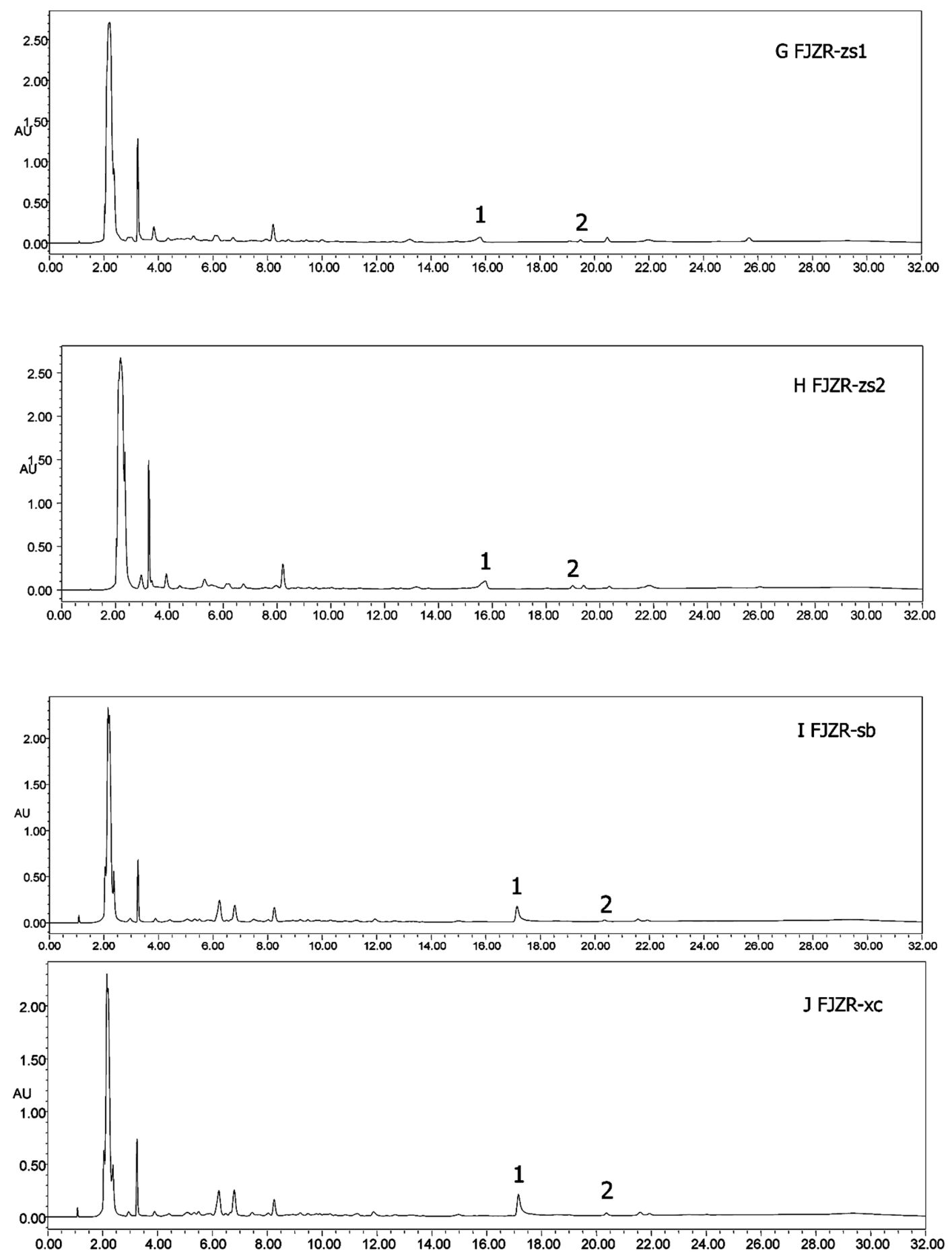

Figure 6. HPLC chromatograms of all samples (A-J). (1) Heterophyllin B, (2) heterophyllin A.

\section{Materials and Methods}

\subsection{Chemicals and Reagents}

Heterophyllin A (1) and Heterophyllin B (2) (Figure 7) were provided by Professor Ninghua Tan (Kunming institute of botany, Chinese academy of science, Kunming, China). HPLC analysis showed that their purities were over 98\%. Analytical grade methanol and acetonitrile were purchased from Sigma (St. Louis, MO, USA). $\mathrm{D}_{2} \mathrm{O}$ was bought from Norell (Landisville, NJ, USA). Sodium 3-trimethlysilyl $\left[2,2,3,3-\mathrm{d}_{4}\right]$ propionate (TSP) was obtained from Cambridge Isotope 
Laboratories, Inc. (Andover, MA, USA). $\mathrm{KH}_{2} \mathrm{PO}_{4}$ was from Beijing Chemical Works (Beijing, China), and $\mathrm{NaOD}$ was purchased from Armar (Dottingen, Switzerland).

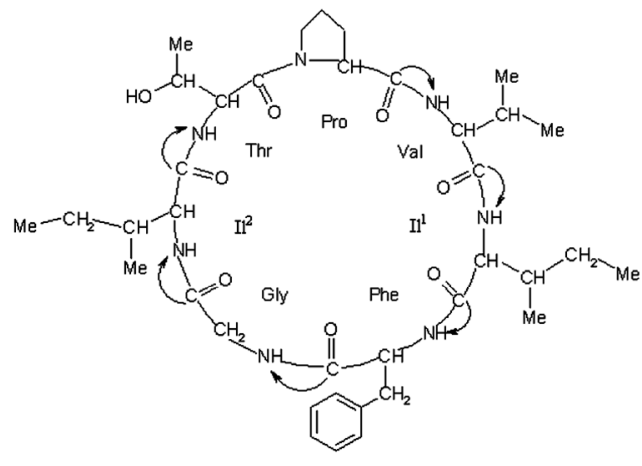

Heterophyllin A (1)

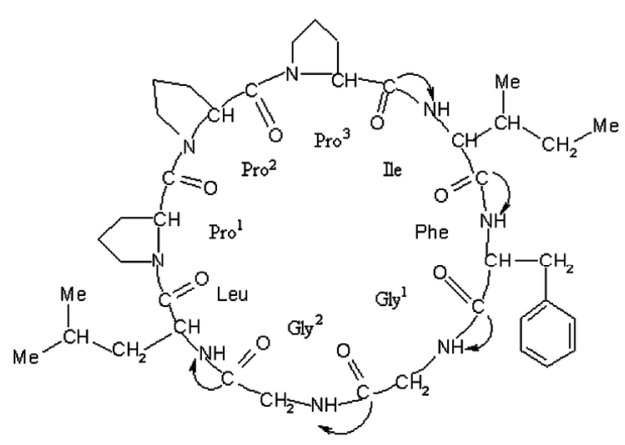

Heterophyllin B (2)

Figure 7. Chemical structures of the two compounds analyzed in the study.

\subsection{Plant Materials}

Five germplasm resources of PR (jr, zs1, zs2, sb, xc) were collected from genuine producing areas (Jurong City, Jiangsu Province; Zherong City, Fujian Province; Shibing City, Guizhou Province; and Xuanzhou City, Anhui Province), and then cultivated in two cultivated bases from Jurong City, Jiangsu Province (JSJR) $\left(119^{\circ} 16^{\prime} 25^{\prime \prime} \mathrm{N}, 31^{\circ} 41^{\prime} 15^{\prime \prime}\right.$ E) and Zherong City, Fujian Province (FJZR) $\left(119^{\circ} 54^{\prime} 2^{\prime \prime} \mathrm{N}\right.$, $27^{\circ} 13^{\prime} 48^{\prime \prime} \mathrm{E}$ ) simultaneously in early November 2012. These samples were harvested in June to August 2013 and were numbered as JSJR-jr, JSJR-zs1, JSJR-zs2, JSJR-sb, JSJR-xc, FJZR-jr, FJZR-zs1, FJZR-zs2, FJZR-sb, FJZR-xc. The samples were authenticated by Prof. Xunhong Liu of Nanjing University of Chinese Medicine. All voucher specimens were deposited in laboratory of identification of Chinese medicine. All samples were ground to a fine powder and sieved with a bolt ( 80 mesh). The powder was then kept in an airtight container at $-80^{\circ} \mathrm{C}$ until use.

\section{3. ${ }^{1} H$-NMR Measurement}

For ${ }^{1} \mathrm{H}-\mathrm{NMR}$ analysis, all powder samples (100 mg each) were transferred into centrifuge tubes. Methanol $(75 \%, 0.6 \mathrm{~mL}$, water:methanol $=1: 2, v / v)$ was added to the tube, followed by vortexing for $1 \mathrm{~min}$ and ultrasonication for $30 \mathrm{~min}$. The materials were then centrifuged at $3500 \mathrm{rpm}$ for $25 \mathrm{~min}$. The supernatants were transferred separately into a $25-\mathrm{mL}$ round-bottomed flask to dry in a rotary vacuum evaporator. The extraction procedure was repeated three times. Samples were dissolved in $600 \mu \mathrm{L}$ mixure (1:1) of $\mathrm{CD}_{3} \mathrm{OD}$ and $\mathrm{KH}_{2} \mathrm{PO}_{4}$ buffer in $\mathrm{D}_{2} \mathrm{O}$ (adjusted to $\mathrm{pH} 6.0$ by $1 \mathrm{~N} \mathrm{NaOD}$ ) containing $0.05 \%$ TSP. The samples were then centrifuged for $10 \mathrm{~min}$ at $13,000 \mathrm{rpm}$, and supernatants $(550 \mu \mathrm{L})$ were transferred into 5-mm tubes for NMR analysis.

${ }^{1} \mathrm{H}-\mathrm{NMR}$ was recorded at $25^{\circ} \mathrm{C}$ on a Bruker $600 \mathrm{MHz}$ AVANCE III NMR spectrometer (Bruker, Karlsruhe, Germany) operating at a proton NMR frequency of $599.83 \mathrm{MHz}$. $\mathrm{CD}_{3} \mathrm{OD}$ was used for internal lock purposes. Each ${ }^{1} \mathrm{H}-\mathrm{NMR}$ spectra consisted of 128 scans requiring $10 \mathrm{~min}$ acquisition time with following parameters: $0.16 \mathrm{~Hz} /$ point, pulse width $(\mathrm{PW})=30^{\circ}(11.3 \mu \mathrm{s})$, and relaxation delay $(\mathrm{RD})=2 \mathrm{~s}$. A presaturation sequence was used to suppress the residual $\mathrm{H}_{2} \mathrm{O}$ signal with low-power selective irradiation at the $\mathrm{H}_{2} \mathrm{O}$ frequency during the recycle delay. Free induction decays (FIDs) were Fourier transformed with Luria-Bertani $(\mathrm{LB})=0.3$. The resulting spectra were manually phased and baseline-corrected, and calibrated to TSP at $0.00 \mathrm{ppm}$. The metabolite data were deposited to MetaboLights [21] with identifier MTBLS399. 


\subsection{Data Analysis}

The ${ }^{1} \mathrm{H}-\mathrm{NMR}$ spectra were processed using MestReNova software (Version 6.1, Mestrelab Research, Santiago de Compostela, Spain), the FID signal was made by Fourier transformation to improve the signal to noise ratio, spectral intensities were scaled to TSP and reduced to integrated regions of equal width $(0.002 \mathrm{ppm})$ according to the region of $\delta 0.50-10.00$. The two regions of $\delta=3.355-3.363$ and 4.67-4.98 were excluded from analysis because of the residual signals of methanol and water. The integral data ( $\delta=0.50$ to $\delta=10.00$, except for $\delta=3.355-3.363$ and 4.67-4.98) were normalized according to peak areas and imported to Microsoft office Excel (Redmond, WA, USA) for the following analysis. The integral data obtained were imported to SIMCA-P 11.5 (Umetrics, Umea, Sweden) for multivariate statistical analysis, including principal component analysis (PCA), partial least squares discriminant analysis (PLS-DA), and orthogonal PLS-DA (OPLS-DA) with VIP $>1$.

\subsection{Content Determination}

All PR samples were weighed exactly to $0.0001 \mathrm{~g}$, and each sample was repeated three times. The content determination of HA and HB in PR was referred to the reliable method developed by our research group [7].

\section{Conclusions}

In this study, we first conducted the metabolic profiling using NMR for the analysis of PR. A total of 34 metabolites were identified based on ${ }^{1} \mathrm{H}-\mathrm{NMR}$ data, and 14 of them were significantly different in PR from different cultivated fields. The relative contents of alanine, lactate, lysine, taurine, sucrose, tyrosine, linolenic acid, $\gamma$-aminobutyrate, and hyperoside in PR from JSJR were higher than that in PR from FJZR, while PR from FJZR contained higher levels of glutamine, raffinose, xylose, unsaturated fatty acid, and formic acid. The contents of HA and HB were higher in PR from FJZR.

The chemical components in metabolomics of TCM are very complex, and the heterophyllins have not been detected in this study. In the further experiment, "add standard qualitative test" will be used to explore the complete assignment and complex chemical components in metabolomics with other analytical techniques. This study will provide the basic information for exploring the influence law of the ecological environment and germplasm genetic variation on metabolite biosynthesis of Pseudostellariae Radix and its quality formation mechanism.

Acknowledgments: This work was supported by the National Natural Science Foundation of China (Award No. 81274016; 81473312) and Jiangsu University Advantage Disciplines Construction Engineering Projects (Award ysxk-2014).

Author Contributions: Yujiao Hua and Ya Hou carried out the experiments, analyzed data and composed the draft of the manuscript. Ya Hou, Yujiao Hua, Shengnan Wang, and Lisi Zou analyzed data and wrote the manuscript. Yang Ma, Lisi Zou, and Zixiu Liu provided the samples of PR. Xunhong Liu participated in the supervision of this study and edited the manuscript. Yiyuan Luo and Juanxiu Liu proposed the study and helped to perform the experiments. All authors read and approved the final manuscript.

Conflicts of Interest: The authors declare no conflict of interest.

\section{References}

1. Liu, X.H.; Han, L.; Wang, L.J; Fu, X.S. Study on Quality Standards of Pseudostellaria heterophylla. Chin. Pharm. 2010, 21, 1769-1771.

2. Pang, W.; Lin, S.; Dai, Q.; Zhang, H.; Hu, J. Antitussive activity of Pseudostellaria heterophylla (Miq.) Pax extracts and improvement in lung function via adjustment of multi-cytokine levels. Molecules 2011, 16, 3360-3370. [CrossRef] [PubMed]

3. Shen, X.C.; Tao, L.; Bo, S.; Gan, H.R.; Duan, J.A. A meliorated effects of Radix Pseudostellariaeon oxidative stress in rat chronic heart failure induced by acute cardiac infarction. West China J. Pharm. Sci. 2008, 23, $413-416$. 
4. Fu, X.S.; Liu, X.H.; Xu, H.; Zhou, Y.Z.; Chen, F. Effect of pseudostellaria polysaccharides in diabetic mice by alloxan. Anhui Med. Pharm. J. 2010, 14, 521-522.

5. Fu, X.S.; Liu, X.H.; Xu, H.; Zhou, Y.Z.; Chen, F. Research status and trends of pseudostellariae radix. Chin. J. New Grugs. 2012, 21, 757-760.

6. Sheng, R.; Xu, X.; Tang, Q.; Bian, D.; Li, Y.; Qian, C.; He, X.; Gao, X.; Pan, R.; Wang, C.; et al. Polysaccharide of radix pseudostellariae improves chronic fatigue syndrome induced by poly I:C in mice. Evid. Based Complement. Altern. Med. 2011, 2011, 840516. [CrossRef] [PubMed]

7. Zou, L.S.; Fu, X.S.; Liu, X.H.; Chao, J.G.; Gu, W.; Ma, Y.; Hou, Y.; Li, Y.R. Study on the dynamic change of Heterophyllin A and B in Radix Pseudostellariae. J. Nanjing Univ. Tradit. Chin. Med. 2013, 29, 175-178.

8. Song, J.P.; Zeng, Y.P.; Liu, X.H.; Zhang, Y.C. Determination of Polysaccharide in Radix Pseudostellariae from different habitats by HPLC-ELSD. Tsinghua J. Tradit. Chin. Med. 2008, 42, 77-79.

9. Fu, X.S.; Zou, L.S.; Liu, X.H.; Ju, W.Z.; Ma, Y.; Hou, Y.; Li, Y.R. Analysis of cyclic peptides in Pseudostellariae Radix by UPLC-ESI-TOF MS/MS. J. Chin. Mass Spectrom. Soc. 2013, 34, 179-184.

10. Ma, Y.; Hou, Y.; Zou, L.S.; Liu, X.H.; Xu, L.; Yuan, J.D. Dynamic changes of nucleosides and nucleobases in Pseudostellariae Radix from Fujian province analyzed by QTRAP LC-MS/MS. Chin. J. New Drugs 2014, 23, 2325-2330.

11. An, K.; He, J.; Wan, Z.M.; Shao, H.W.; Yang, X.C.; Jiao, W. Determination and multivariate statistical analysis of amino acid in Pseudostellaria Heterophylla from different producing areas. Nat. Prod. Res. Dev. 2012, 594-598.

12. Li, Z.Y.; Zhi, H.J.; Zhang, F.S.; Sun, H.F.; Zhang, L.Z.; Jia, J.P.; Xing, J.; Qin, X.M. Metabolomic profiling of the antitussive and expectorant plant Tussilago farfara L. by nuclear magnetic resonance spectroscopy and multivariate data analysis. J. Pharm. Biomed. Anal. 2013, 75, 158-164. [CrossRef] [PubMed]

13. Jia, J.; Zhang, F.; Li, Z.; Qin, X.; Zhang, L. Comparison of fruits of forsythia suspensa at two different maturation stages by NMR-based metabolomics. Molecules 2015, 20, 10065-10081. [CrossRef] [PubMed]

14. Fan, G.; Tao, L.H.; Yue, Q.H.; Kuang, T.T.; Tang, C.; Yang, Y.D.; Luo, W.Z.; Zhou, X.D.; Zhang, Y. Metabolic discrimination of rhizoma coptidis from different species using ${ }^{1} \mathrm{H}-\mathrm{NMR}$ spectroscopy and principal component analysis. Planta Med. 2012, 78, 641-648. [CrossRef] [PubMed]

15. Yang, S.O.; Shin, Y.S.; Hyun, S.H.; Cho, S.; Bang, K.H.; Lee, D.; Choi, S.P.; Choi, H.K. NMR-based metabolic profiling and differentiation of ginseng roots according to cultivation ages. J. Pharm. Biomed. Anal. 2012, 58, 19-26. [CrossRef] [PubMed]

16. Chatterjee, S.; Srivastava, S.; Khalid, A.; Singh, N.; Sangwan, R.S.; Sidhu, O.P.; Roy, R.; Khetrapal, C.L.; Tuli, R. Comprehensive metabolic fingerprinting of Withania somnifera leaf and root extracts. Phytochemistry 2010, 71, 1085-1094. [CrossRef] [PubMed]

17. Kim, H.K.; Choi, Y.H.; Verpoorte, R. NMR-based metabolomic analysis of plants. Nat. Protoc. 2010, 5, 536-549. [CrossRef] [PubMed]

18. Ben-Izhak Monselise, E.; Parola, A.H.; Kost, D. Low-frequency electromagnetic fields induce a stress effect upon higher plants, as evident by the universal stress signal, alanine. Biochem. Biophys. Res. Commun. 2003, 302, 427-434. [CrossRef]

19. Tauzin, A.S.; Giardina, T. Sucrose and invertases, a part of the plant defense response to the biotic stresses. Front. Plant Sci. 2014, 5. [CrossRef] [PubMed]

20. Zhang, C.F.; Tian, S.P. Peach fruit acquired tolerance to low temperature stress by accumulation of linolenic acid and $N$-acylphosphatidylethanolamine in plasma membrane. Food Chem. 2010, 120, 864-872. [CrossRef]

21. Alisdair, R.F.; Asaph, A.; Lothar, W.; Mark, S.; Takayuki, T.; Joachim, K.; Adam, J.C.; Kazuki, S.; Paul, D.F.; Vincenzo, D. Recommendations for reporting metabolite data. Plant Cell 2011, 23, 2477-2482.

Sample Availability: Samples of the compounds $\mathbf{1}$ and $\mathbf{2}$ are available from the authors. 